\title{
Dynamics of the extremely elongated cloud on Mars Arsia Mons volcano
}

Jorge Hernández-Bernal (1), Agustín Sánchez-Lavega(1), Teresa del Río-Gaztelurrutia (1), Ricardo Hueso (1), Alejandro Cardesín-Moinelo (2), Eleni M. Ravanis (2), D.Titov (3), Simon Wood (4), K. Connour (5), N. Schneider (5), B. Gondet (6), D. Tirsch (7), R. Jaumann (7), E. Hauber (7)

(1) Dpto. Fisica Aplicada I, Escuela de Ingeniería de Bilbao, UPV/EHU, Bilbao, Spain, (2) European Space Agency, ESAC, Madrid, Spain, (3) European Space Agency, ESTEC, Noordwijk, Netherlands, (4) European Space Agency, ESOC, Darmstadt, Germany, (5) Laboratory for Atmospheric and Space Physics, University of Colorado, Boulder, USA, (6) Institut d'Astrophysique Spatiale, CNRS/University Paris Sud, Orsay, France, (7) German Aerospace Center (DLR), Institute of Planetary Research, Rutherfordstr. 2, 12489 Berlin, Germany (Jorge.hernandez@ehu.eus)

\begin{abstract}
Starting on 13 September 2018, an extremely elongated cloud rising from the Arsia Mons volcano was spotted during at least 80 sols by the VMC camera onboard Mars Express [1]. A similar cloud was found in the VMC archive in different Martian Years (MY), around the southern solstice (Ls $270^{\circ}$ ), a season known for a general lack of clouds in the planet [2]. The cloud reached longitudes of more than $1500 \mathrm{~km}$, altitudes of around $30 \mathrm{~km}$, and growth velocities faster than $100 \mathrm{~m} / \mathrm{s}$ when expansion of its tail could be observed. A high variability of the cloud depending on the local time is observed. Interannual variations are also discussed, including its relation with the 2018 Global Dust Storm (GDS 2018).
\end{abstract}

\section{Introduction}

The season before the Martian southern solstice is known for the general lack of water ice clouds on the planet, however, the Arsia Mons volcano seems to be an exception to this, as orographic clouds appear around it in this season [2]. The VMC camera onboard Mars Express is an engineering camera upgraded to science instrument [1] that mostly takes low resolution full-disk images of Mars, providing coverage of large areas of the planet at a wide range of local times [3,4]. This particular coverage enabled VMC to reveal an impressive elongated cloud since September 2018, which is before the southern solstice, and following the decline of the GDS 2018 [5], appearing at the western slope of the Arsia Mons volcano in the sunrise, and reaching more than 1500 $\mathrm{km}$ in length during the following hours. The cloud appeared everyday during at least 80 sols in Martian Year 34 (2018), and was observed by VMC, HRSC, and OMEGA onboard Mars Express, and IUVS onboard MAVEN. We study the behavior of this singular cloud depending on the local time, the interannual variations in its apparition every Martian year, and its possible value as a proxy for studying interannual variations in the climate of Mars.

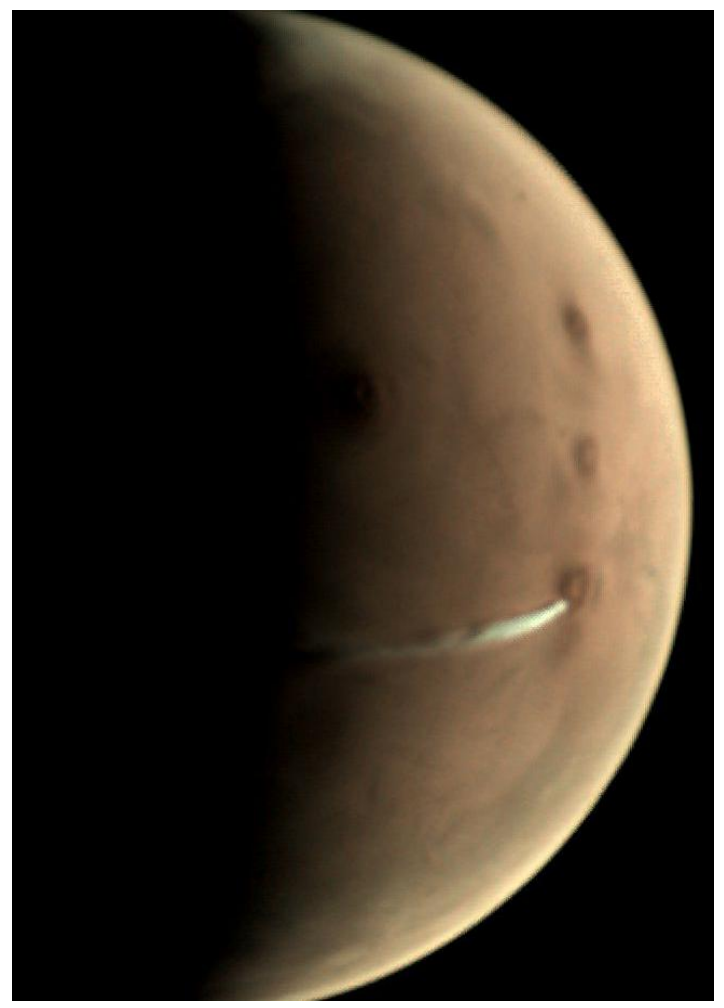

Figure 1: The Arsia Mons volcano in the Tharsis region and its elongated cloud extending more than a thousand kilometers westward from the volcano in the first hours of the morning. Captured by MEx/VMC on October 10, 2018. 


\section{The dataset}

The MEx/VMC dataset includes around 30 observations related to the cloud in 2018, containing around 1000 images. Some observations lasted several minutes (up to 80 minutes), enabling cloud tracking of the cloud dynamics. We examined the VMC archive and found 30 additional VMC observations related to similar clouds on Arsia Mons in the same season in previous years.

MAVEN/IUVS took several images of the cloud at different dates. IUVS images are obtained simultaneously with 19 filters and are calibrated in reflectivity. MEx/OMEGA and MEx/HRSC are high resolution cameras that provide detailed images of the event, thus their field of view is narrower and they captured the event with less frequency. OMEGA also provides multispectral images.

\section{Behavior with local time}

The most unusual characteristic of this phenomenon is the elongated tail shape of the cloud. However, images from different instruments at different local times show that the shape of the cloud evolves rapidly with time. The cloud grows every morning at the western slope of the Arsia Mons volcano, at this stage, the cloud grows vertically, reaching such altitude (up to $30 \mathrm{~km}$ ) that it can receive direct sunlight illumination before the sunrise [6]. During the first hours of the morning, the cloud grows in the western direction, forming its characteristic elongated tail. By applying the cloud-tracking method, we have found winds faster than $100 \mathrm{~m} / \mathrm{s}$ in this tail. Later, as the insolation increases, the tail dissolves, while in general the core orographic cloud stands around the Arsia Mons volcano, as observed by MRO/MARCI [7] in the midday.

\section{Interannual variations}

The elongated cloud has been observed by different instruments around Southern solstice every Martian year (MY), at least since MY 29. The most complete coverage was performed in MY 34, when it existed at least for 80 sols. Through this period the shape and optical depth of the cloud seemed to evolve.

We study the phenomenon across several years, in search for variations in its Ls range, or any other measurable parameters. This phenomenon might hold the added value of being useful as a proxy for interannual variations in the atmosphere, similar to an already studied cyclone also observed by VMC and other instruments [3].

\section{Acknowledgements}

This work has been supported by the Spanish project AYA2015-65041-P (MINECO/FEDER, UE) and Grupos Gobierno Vasco IT-765-13. JHB was supported by ESA Contract No. 4000118461/16/ES/JD, Scientific Support for Mars Express Visual Monitoring Camera. We acknowledge support from the Faculty of the European Space Astronomy Centre (ESAC)

\section{References}

[1] Sánchez-Lavega, A., et al. "Limb clouds and dust on Mars from images obtained by the Visual Monitoring Camera (VMC) onboard Mars Express." Icarus 299 (2018): 194-205.

[2] Wang, Huiqun, and Andrew P. Ingersoll. "Martian clouds observed by Mars global surveyor Mars orbiter camera." Journal of Geophysical Research: Planets 107.E10 (2002): 8-1.

[3] Sánchez-Lavega, A., et al. "A seasonally recurrent annular cyclone in Mars northern latitudes and observations of a companion vortex." Journal of Geophysical Research: Planets 123.11 (2018): 3020-3034

[4] Hernández-Bernal, J. et al. "The 2018 Martian Global Dust Storm over the South Polar Region studied with $\mathrm{MEx} / \mathrm{VMC}$ " (in preparation)

[5]Sánchez-Lavega A. et al.: "The onset and growth of the 2018 Martian Global Dust Storm" Geophus. R. Lett., submitted, 2019

[6] Hernández-Bernal, J. et al. "Clouds in the night side of Mars: an analysis using Mars Express VMC", EPSC2018, 16-21 September 2018, Berlin, Germany.

[7] MRO MARCI Mars Weather Report for the week of 8 october - 14 october 2018

http://www.msss.com/msss_images/2018/10/17/ 